\title{
A Fast and Accurate Computation of Interconnect Capacitance
}

\author{
S. Putot, F. Charlet, P. Witomski* \\ LETI (CEA-Technologies Avancées), 17 rue des Martyrs, 38054 Grenoble Cedex 09, FRANCE \\ phone : 33476884738 fax : 33476889457 email : sputot@cea.fr \\ *LMC-IMAG, BP 53, 38041 Grenoble Cedex 9, FRANCE
}

\begin{abstract}
We present a new simulation tool for efficient extraction of $3 \mathrm{D}$ capacitance matrix in interconnect structures embedded in a multilayered dielectric environment. We use a fictitious domain method, which replaces the initial problem on a complex geometry by a problem on a simple shape domain embedding the initial domain, and is consequently ideally suited for the treatment of complex geometries. Numerical results confirm that this method is more efficient, both in cpu time and memory, than a finite elements or a boundary elements method.
\end{abstract}

\section{Introduction}

The capacitance are calculated from the distribution of charge density on the surfaces of the conductors. Classically, two approaches are used to determine the charge density.

The problem can be formulated as a volume partial differential equation on the potential, which can be solved using finite difference or finite element methods. The charges are then got by the normal derivative of the potential on the surfaces. The resultant matrix is sparse but large because the whole dielectric volume is discretized. Non-homogeneous media and arbitrary conductor shapes can be handled. However, mesh generation is a critical point.

Alternatively, an integral equation formulation can be used, leading to a smaller system, since only the surfaces are discretized. The solution of this system gives directly the surface charges. But this system is dense and the handling of multidielectric media is not natural, and very time and memory consuming.

The method we propose combines advantages of volume methods and integral equations methods. The meshing of a complex 3D domain is avoided by the use of two meshes, a regular $3 \mathrm{D}$ grid on a simple shape domain embedding the initial domain, and a surface mesh of the conductors. Multilayered media are taken into account at no extra cost, and the formulation leads to a sparse system. Moreover, thanks to the regularity of the volume grid, fast solvers can be used for the solution of this system and little storage is required.

In the first paragraph, we introduce the so-called fictitious domain method with Lagrange multipliers for the problem formulation. It is based on (1), and has been introduced in the context of interconnect structures in (2). Then in the second paragraph, we discuss the approximation of the potential and charge in finite dimensional spaces, and the solution of the resulting system : in (1), the variable of interest is the potential, whereas here we improved the method to get a good accuracy on the charge too. Numerical results then confirm both the gain of accuracy on the charge compared to a standard approximation of the fictitious domain formulation, and the efficiency of the algorithm obtained, compared to a finite elements or a boundary elements method.

\section{The Fictitious Domain Formulation}

The main idea of fictitious domain methods is to replace the problem on a domain of complex geometry by another one on a simple shape domain, the fictitious domain (Fig. 1), thus allowing the use of a regular grid of that domain and of fast solvers. Here the potential computation is artificially extended inside the conductors, and the boundary condition is taken into account via the introduction of a Lagrange multiplier $\lambda$ defined on the surface mesh, that can be interpreted as the surface charge. Considering the boundary conditions on the conductors surfaces as equality constraints, the problem can be reformulated as the saddle-point problem associated to a constrained minimization problem. This leads to a coupled system, which unknowns are the potential on the fictitious domain, and the charge on the conductors surfaces.

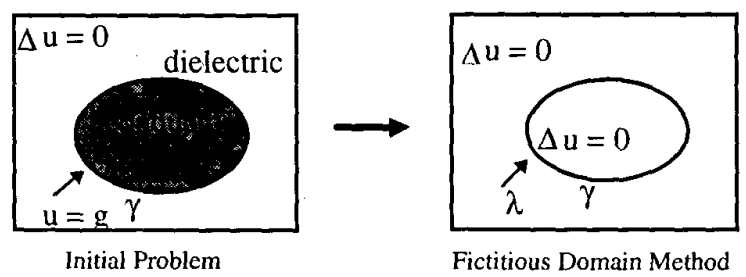

Fig. 1 : Principle of Fictitious Domain Methods

Let $\omega$ the dielectric space between the conductors, of permittivity $\varepsilon$, and $\gamma$ the union of the conductor surfaces. We note $\Omega$ the set constituted by the dielectric regions and the conductors, and $\Gamma$ the boundary of the fictitious domain $\Omega$ (Fig. 2). The potential $u$ in $\omega$ is the solution of
(P) $\left\{\begin{array}{l}\nabla(\varepsilon \nabla u)=0 \text { in } \omega \\ u=g \text { on } \gamma \\ + \text { boundary conditions on } \Gamma\end{array}\right.$ 


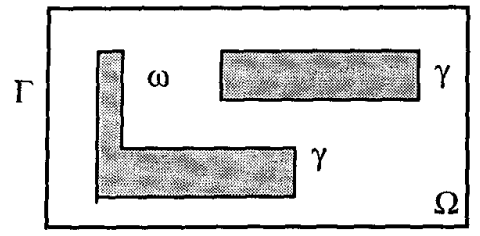

Fig. 2: Notations

The potential can also be seen as the restriction to $\omega$ of the solution $\tilde{u}$ of the extended minimization problem

$$
\min _{v_{\gamma}=g} \frac{1}{2} \int_{\Omega} \varepsilon|\nabla v|^{2} d x \text {, where } v \text { defined in } \Omega \text { verifies (P.c). }
$$

We introduce the Lagrangian associated to that problem by

$$
L(v, \mu)=\frac{1}{2} \int_{\Omega} \varepsilon|\nabla v|^{2} d x-\int_{\gamma} \mu(v-g) d \gamma .
$$

Then the extended potential is the first term of the saddlepoint $(\tilde{u}, \lambda) \in X \times M$ solution of

$$
\text { (Q) }\left\{\begin{array}{l}
\int_{\Omega} \varepsilon \nabla \tilde{u} \nabla v d x=\int_{\gamma} \lambda v d \gamma \quad \forall v \in X \\
\int_{\gamma} \mu(\tilde{u}-g) d \gamma=0 \quad \forall \mu \in M
\end{array}\right.
$$

where $\mathrm{X}$ and $\mathrm{M}$ are proper spaces of functions.

The potential is the restriction of $\tilde{u}$ to the dielectric region $\omega$, and the charge is equal to $\lambda$ on the surface $\gamma$.

\section{Discretization and Solution}

In comparison with the standard approximation, the originality of our approach consists in decomposing the potential $\tilde{u}$ on the fictitious domain $\Omega$ by

$$
\tilde{u}=u^{r}+u^{n r},
$$

where $u^{r}$ is the regular part, which we approach using $Q_{1}$ -

finite elements on a regular grid of parallelepipeds, and $u^{n r}$ is the irregular part, due to the jump of the normal derivative of the potential on the conductor surfaces $\gamma$. This part is approached using non regular functions $\Phi_{K_{i}}$, defined on parallelepipeds $K_{i}$ that have a non-zero intersection with $\gamma$ (see Fig. 3-4). The potential is thus approached by a vector

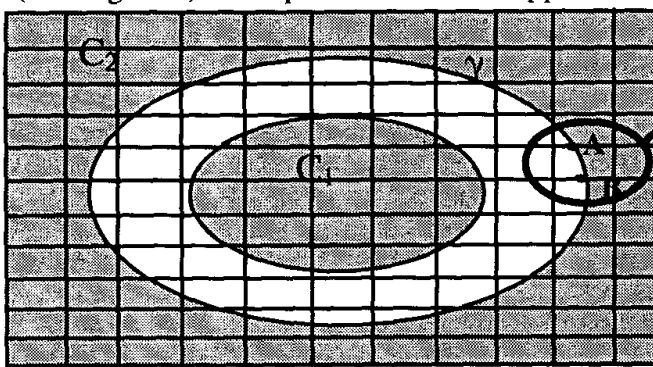

Fig. $3: 2 \mathrm{D}$ test case (on which we show the accuracy of the method in Fig.5)
$(U, V)$, where $U$ is its decomposition on the $Q_{1}$-finite clements basis, and $V$ on the functions $\Phi_{K_{i}}$.

The charge $\lambda$ is approached by $\Lambda$, using $P_{0}$-finite elements on a mesh of the surface $\gamma$. With our choice for the functions $\Phi_{K_{i}}$ (see Fig. 4), and in the case when the surfaces of conductors are parallel to the grid, the discrete formulation of system $(Q)$ allows us to express $V$ as a function of $U$ and $\Lambda$. The vectors $U$ and $\Lambda$ are then the solution of

$$
\left(Q_{h}\right) \quad\left\{\begin{array}{l}
A U-B \Lambda=0 \\
B^{T} U+C \Lambda=g
\end{array}\right.
$$

The same formulation has been programmed even when the conductors surfaces are not parallel to the grid mesh. It is known that a saddle-point problem must satisfy a condition known as the inf-sup condition (5) to ensure the existence of a solution. In (6) they prove that with the standard approximation of the potential by $Q_{1}$-finite elements, and of the charge by $P_{0}$-finite elements, the convergence of the: method requires that the grid must not be too coarse compared to the surface mesh. However, numerical results show that with our approximation, this condition can be relaxed, and accurate results are obtained even for coarse grids, whether or not the conductor surfaces are parallel to the grid mesh (see e.g. Fig. 5 in 2 D).

This system can be rewritten as a symmetric definite positive system in $\Lambda$,

$$
\left(B^{T} A^{-1} B+C\right) \Lambda=g,
$$

and solved by the conjugate gradient algorithm.

Thanks to the regularity of the 3D mesh, the matrix $A$ doesn't need to be stored, and its inversion is computed with a fast solver using FFT. We remark that a fast Poisson solver (4) takes advantage of the regularity of the equation and grid only in 2 directions. This allows us to adapt the solver to the case of dielectric layers without extra cost. Moreover, $C$ is diagonal and $B$ sparse $(\approx 20 m$ elements stored, with $m$ the number of nodes on the surface mesh). Indeed $B$ represents the coupling between the surface mesh and the grid.

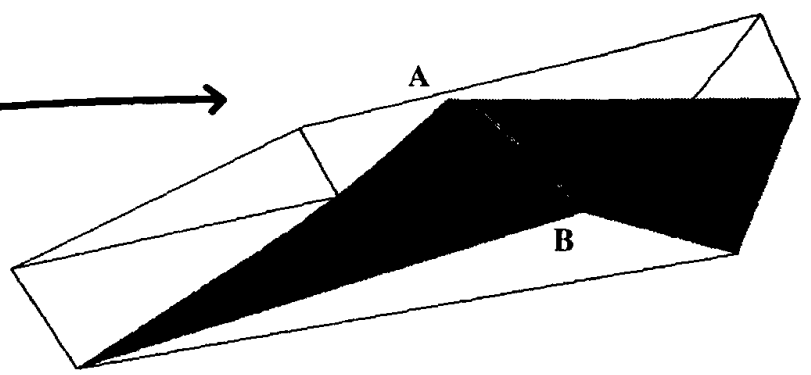

Fig. 4 : 2D example of the shape function $\Phi_{K_{i}}$ on a rectangle : zero at the nodes of the rectangle, with a jump of normal derivative on the surface $\gamma$. 


\section{Results}

We first show on a $2 \mathrm{D}$ test case (Fig. 3) the accuracy of our approximation compared to the classical approximation that approaches only the regular part of the potential, then compare on complex 3D structures the efficiency of the method with other methods.

Our improvement of the approximation leads to a significant gain in accuracy of the charge when the volume grid is coarse (Fig. 5). This is important as the cpu time (and memory) depends almost linearly on the number of nodes on the volume grid, while it is not very sensitive to the surface mesh refinement (Fig. 6). In this example, an accuracy of $1 \%$ is obtained with our approximation for only 200 grid points and $0.05 \mathrm{cpu}$ seconds, while the same accuracy requires $10^{5}$ points and 2 seconds with the standard approximation.

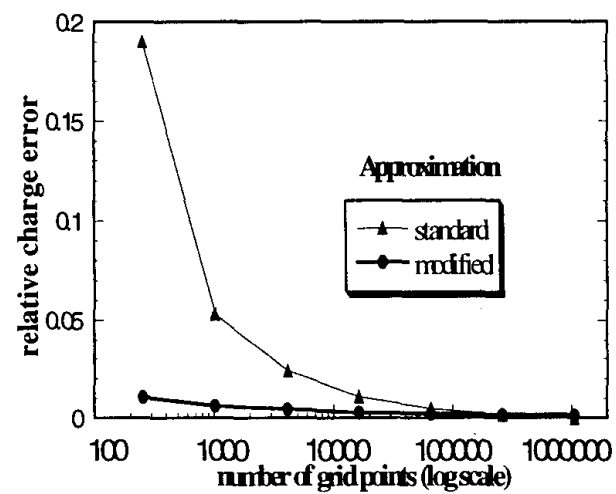

Fig. 5 : Comparison of the standard/modified approximations on the $2 \mathrm{D}$ test problem of Fig. 3

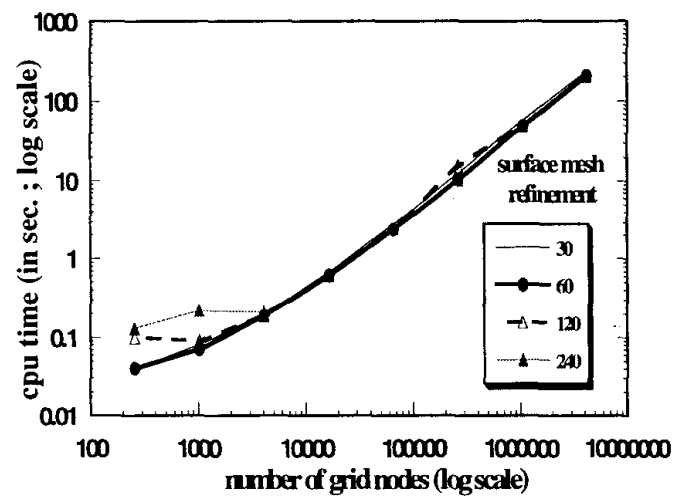

Fig. 6 : Cpu time for both approximations on the $2 \mathrm{D}$ test problem of Fig. 3
Then we present complex structures, with several levels of metallization and dielectrics (cel_3 - Fig. 7, cel_1 - that looks like cel_3 but smaller), and one (cel_2 - Fig. 8) that has particularities (length very large compared to the width and height of lines, influence of the ground plane much stronger than mutual influence of conductors) that make the computation difficult. We show on the example of the structure cel_3 that the volume mesh doesn't have to be very refined compared to the conductor shapes: in Fig. 9 is represented the volume mesh used for the computations. For the surface mesh, we used the intersection of the conductor surfaces and the volume grid. The results for these three structures (Table 1, Fig. 10) confirm the efficiency of the method compared to finite elements (SILVACO's Clever) or boundary elements methods (MIT's Fastcap).

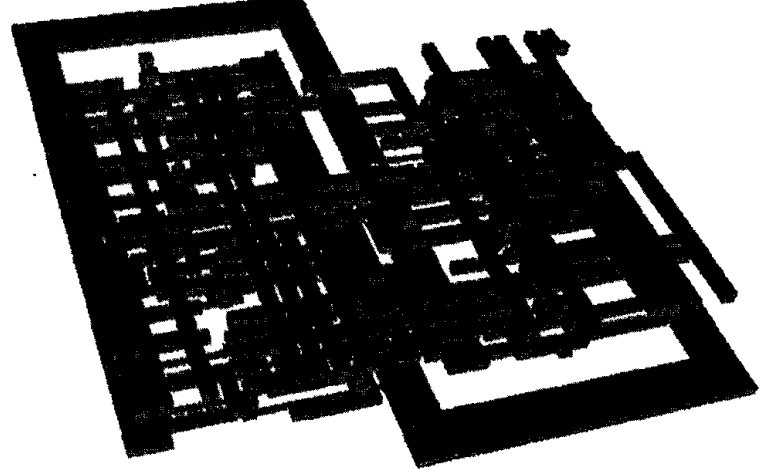

Fig. 7: cel_3 (last example of Table 1)

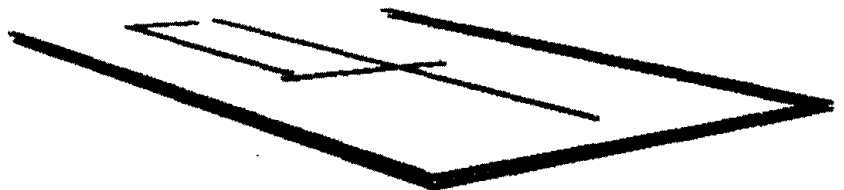

Fig. 8 : cel_2. Note that the lengths are very large compared to the width or height of a line.

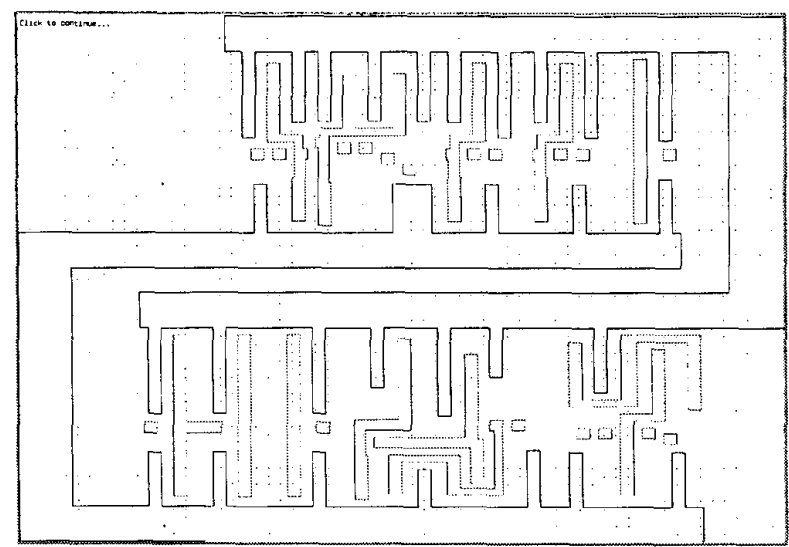

Fig. 9 : Horizontal cut of structure cel_3. The points are the nodes of the volume grid used for computation, the conductors are represented. 

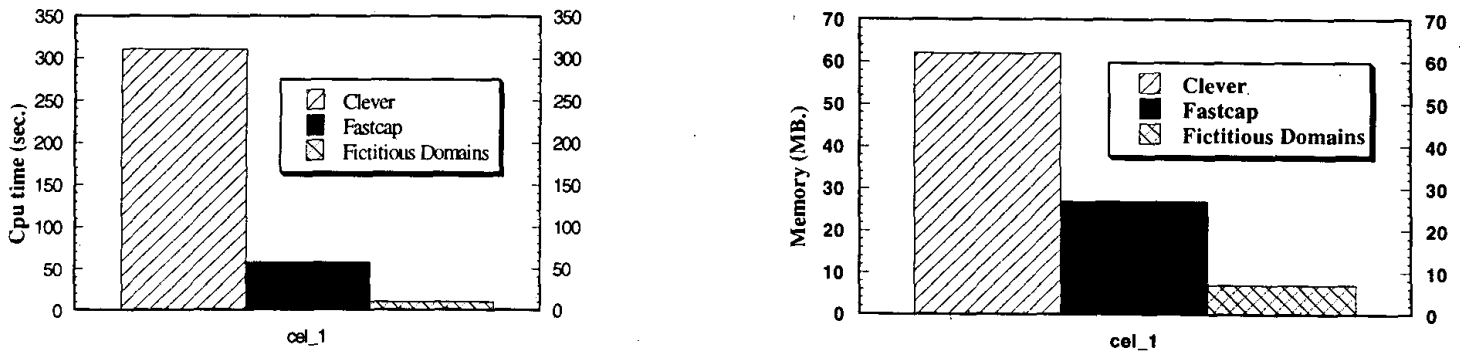

Fig. 10: Cpu time and Memory used by the 3 methods on the 3D structure cel_1.

Table 1 : Comparisons of the fictitious domain method with a finite elements method (SLVACO's Clever) and'a boundary elements method (MIT's Fastcap) on 3D structures (Fig. 7 and 8), for same precision criterium of 5\%. The values compared are the largest capacitance for cel_1 and cel_3, and th: mutual capacitance between the two electrodes for cel_2. Note also that we used an homogeneous dielectric for Fastcap simulations, therefore its cpu ani memory results are optimistic, and we can't fully compare the values.

\begin{tabular}{|c|c|c|c|c|c|}
\hline & & Clever & Fastcap & Fictitious Dom. & Measurements \\
\hline \multirow{3}{*}{$\begin{array}{l}\text { cel_1 } \\
20 \mu \mathrm{m} \times 20 \mu \mathrm{m} \\
3 \text { levels, } 2 \text { dielectrics }\end{array}$} & Values (F) & $1.55 \mathrm{e}^{-14}$ & & $1.41 \mathrm{e}^{-14}$ & \\
\hline & Time (sec) & 311 & 58 & 10 & \\
\hline & Memory (MB) & 62 & 27 & 7 & \\
\hline \multirow{3}{*}{$\begin{array}{l}\text { cel_2 } \\
125 \mu \mathrm{m} \times 150 \mu \mathrm{m} \\
2 \text { levels, } 4 \text { dielectrics }\end{array}$} & Values $(F)$ & $4.67 \mathrm{e}^{.16}$ & & $7.8 e^{-16}$ & \multirow[t]{3}{*}{$6.88 \mathrm{e}^{-16} \pm 1.4 \mathrm{e}^{-16}$} \\
\hline & Time (sec) & 1507 & 17 & 12 & \\
\hline & Memory (MB) & 387 & 18 & 5.5 & \\
\hline \multirow{3}{*}{$\begin{array}{l}\text { cel_3 } \\
35 \mu \mathrm{m} \times 30 \mu \mathrm{m} \\
5 \text { levels, } 3 \text { dielectrics }\end{array}$} & Values (F) & $2.64 \mathrm{e}^{-16}$ & & $2.43 \mathrm{e}^{-16}$ & \\
\hline & Time (sec) & 1389 & 500 & 40 & \\
\hline & Memory (MB) & 339 & 443 & 23 & \\
\hline
\end{tabular}

\section{Conclusion}

Fictitious domain methods, based on the idea of replacing a problem on a complex geometry by another problem on a simple shape domain embedding the initial domain of computation, seem ideally suited to the treatment of interconnect structures, which have complicated threedimensional shapes. The particular formulation presented here for the determination of capacitance has the advantage of giving directly not only the potential, but also the surface charge. Our improvement of the approximation compared to a classical approximation leads to accurate results even for coarse grids. Thanks to the use of a regular grid of the embedding domain, the meshing of a complex threedimensional domain is avoided, and fast solvers can be used. The algorithm thus obtained has proved very efficient both in cpu time and memory for static capacitance calculations.

Moreover, similar methods using a regular volume grid can be applied to the extraction of parasitic effects in the frequency domain (calculation of the resistance, capacitance, inductance and conductance as a function of the frequency).

\section{Acknowledgments}

This work has been carried out in the frame of GRESSI consortium between CEA.G-LETI and FRANCE TELECOMCNET.

\section{References}

(1) R. Glowinski,, T.W. Pan, J. Periaux, "A fictitious domain method for Dirichlet problem and applications ", Comp. Meth. Appl. Mech. Eng., vol. 111, 1994, pp.283-303.

(2) S. Putot, F. Charlet, P. Witomski, SISPAD 1999, in press.

(3) K. Nabors and J. White, "Fastcap: a multipole accelerated 3-D capacitance extraction program", IEEE Trans. on Computer-Aided Design of Int. Circ. Syst, vol. 10, pp. 1447-1459, Nov. 91.

(4) N. Swarztrauber, "The methods of cyclic reduction, Fourier analysis and the FACR algorithm for the discrete solution of Poisson's equation on a rectangle ", SIAM Review, vol. 19, no 3, July 1977, pp. 490-501.

(5) F. Brezzi, "On the existence, uniqueness and approximation of saddlepoint problems arising from lagrangian multipliers", R.A.I.R.O., vol. 8, août 1974, 2, pp. 129-151.

(6) V. Girault and R. Glowinski, "Error analysis of a fictitious domain method applied to a Dirichlet problem", Japan J. Indus. Appl. Math., 12(3), 1995, pp. 487-514. 